\title{
miR-217-5p regulates myogenesis in skeletal muscle stem cells by targeting FGFR2
}

\author{
MENGHAI ZHU, GANG CHEN, YI YANG, JIANTAO YANG, BENGANG QIN and LIQIANG GU \\ Department of Orthopedic Trauma and Microsurgery, The First Affiliated Hospital, \\ Sun Yat-sen University, Guangzhou, Guangdong 510080, P.R. China
}

Received December 20, 2019; Accepted April 4, 2020

DOI: $10.3892 / \mathrm{mmr} .2020 .11133$

\begin{abstract}
MicroRNA-217-5p (miR-217-5p) has been implicated in cell proliferation; however, its role in skeletal muscle stem cells (SkMSCs) remains unknown. The present study aimed to explore the roles of miR-217-5p in the biological characteristics of SkMSCs. SkMSCs were identified by cell surface markers using flow cytometry. The present study observed that miR-217-5p mimics accelerated the proliferation and suppressed the differentiation in SkMSCs. In addition, the results of the present study revealed that fibroblast growth factor receptor 2 (FGFR2) was a target of miR-217-5p, as miR-217-5p bound directly to the 3'-untranslated region of FGFR2 mRNA, resulting in increased FGFR2 mRNA and protein levels. In addition, the present study suppressed the expression of FGFR2 in SkMSCs using a selective FGFR inhibitor AZD4547 and detected the efficiency of inhibition by reverse transcription-quantitative PCR and western blotting. miR-217-5p levels were positively associated with FGFR2 expression, which was upregulated and accelerated the proliferation of SkMSCs compared with that of the miR-NC group. Collectively, these results demonstrated that miR-217-5p may act as a myogenesis promoter in SkMSCs by directly targeting FGFR2 and may regulate the myogenesis of these cells.
\end{abstract}

Correspondence to: Dr Bengang Qin or Professor Liqiang Gu, Department of Orthopedic Trauma and Microsurgery, The First Affiliated Hospital, Sun Yat-sen University, 58 Zhongshan 2nd Road, Guangzhou, Guangdong 510080, P.R. China

E-mail: gangxiaxuan@163.com

E-mail: guliqiang1963@aliyun.com

Abbreviations: MyHC, myosin heavy chain; MyoD, myogenic differentiation markers; Pax7, paired box protein7; SkMSCs, skeletal muscle stem cells; TGF $\beta 1$, transforming growth factor- $\beta 1$; EdU, 5-Ethynyl-2'-deoxyuridine; FACS, fluorescence-activated cell sorting; UTR, untranslated region

Key words: fibroblast growth factor receptor 2, microRNA-217-5p, myogenesis, skeletal muscle stem cells

\section{Introduction}

Skeletal muscle stem cells (SkMSCs) are capable of self-renewal and muscle regeneration (1). Lineage progression directs quiescent stem cells toward activation, proliferation and differentiation due to muscle injury or pathological conditions through the activation of multiple mitogenic factors (2-4). Previous studies have suggested that key transcription factors serve a significant role in the proliferation and differentiation of SkMSCs $(4,5)$. Progenitors expressing paired box (Pax) proteins $3 / 7$ are prerequisite factors for skeletal muscle growth and are regarded as the source of adult SkMSCs (5-7). A number of studies have suggested that the expression of Pax7 is important for the maintenance of adult SkMSCs, and that the activation of the myogenic differentiation markers (MyoD) gene primes myogenesis $(6,8,9)$.

Accumulating evidence suggests that a number of microRNAs (miRNAs), such as miR-99a-5p (10), miR-9-5p (11), miR-208b (12), serve important roles in skeletal muscle myogenesis by regulating gene expression, and that their abnormal expression is associated with a number of muscle diseases including, muscle atrophy and ischemic injury $(6,13,14)$. Regarding the molecular mechanism of miRNAs in myogenesis, studies have reported a role for these molecules in the differentiation of SkMSCs $(13,15)$. Indeed, substantial evidence supports the hypothesis that miRNAs are involved in regulating muscle regeneration. A recent study has reported that miR-483-3p is involved in the osteogenic differentiation of bone marrow-derived mesenchymal stem cells (BMSCs) by targeting STAT1 and may serve as a potential therapeutic target for bone loss due to aging (16). In addition, studies have revealed that miR-7 regulates the neural differentiation of trabecular meshwork mesenchymal stem cells (TMMSCs), and that the poly (L-lactate) (PLLA)/poly (e-caprolactone) (PCL) scaffold, termed a three-dimensional (3D) culture system, can promote their differentiation towards glial and neural progenitor cells (14). Previous studies have reported that miRNA-217-5p regulates pluripotent stem cell proliferation and differentiation (17) and is involved in metabolic processes in various cells, such as endothelial (18) and colorectal cancer (19) cells. These findings provide insights into the application of miRNAs in regenerative and cell therapy for muscle diseases (14). However, a limited number of studies have explored the potential roles of miR-217-5p in SkMSCs. 
Previous studies have reported that fibroblast growth factor receptor 2 (FGFR2) exerts an important role in embryogenesis and tissue regeneration, especially in bone and vascular development $(20,21)$. FGFR2 overexpression serves a crucial role in the myogenesis of SkMSCs (22). Consistent with these findings, owing to its association with the myogenesis of SkMSCs, FGFR2 is considered a therapeutic target for muscle injury $(23,24)$. However, its role in the proliferation of SkMSCs remains unclear. This present study aimed to investigate whether miR-217-5p may mediate the expression of FGFR2 in SkMSCs.

\section{Materials and methods}

Animals and cell culture. Isolated single myofiber-associated cells were prepared using limb muscles obtained from 2-week-old female Sprague Dawley rats $(\mathrm{n}=5 ; 30-500 \mathrm{~g})$ maintained in a $12: 12 \mathrm{~h}$ light/dark cycle at $23^{\circ} \mathrm{C}$ and $50-70 \%$ humidity, which were anesthetized with $50 \mathrm{mg} / \mathrm{kg} 1 \%$ sodium pentobarbital and euthanized by cervical dislocation prior to the removal of the limb muscles. Animal experiments were approved by The Institutional Animal Care and Use Committee at The First Affiliated Hospital of Sun Yat-sen University (Guangzhou, China). All animals were purchased from The Guangdong Medical Laboratory Animal Center (Guangzhou, China). Rat tibialis muscles were subjected to enzymatic dissociation $(0.2 \%$ collagenase, Sigma-Aldrich; Merck KGaA) at $37^{\circ} \mathrm{C}$ for $90 \mathrm{~min}$. The cell suspension was filtered through a $40-\mu \mathrm{m}$ filter (Biosharp Life Sciences). Following isolation, myofiber-associated cells were stained for the isolation of particular cell populations by flow cytometry and fluorescence-activated cell sorting (FACS). After sorting, cells were cultured in DMEM (HyClone; GE Healthcare life Sciences) supplemented with 20\% FBS (Gibco; Thermo Fisher Scientific, Inc.) and $1 \%$ chick embryo extract (Gemini Bio Products). Myogenic differentiation was induced using DMEM with 2\% heat-inactivated horse serum (Gibco; Thermo Fisher Scientific, Inc.). The NC group cells were cultured in DMEM supplemented with $10 \%$ FBS and the PG group cells were incubated in DMEM supplemented with $20 \%$ FBS, $1 \%$ chick embryo extract and transforming growth factor (TGF) $\beta 1$. The cells were maintained in an incubator with a humidified atmosphere of $95 \%$ air and $5 \% \mathrm{CO}_{2}$ at $37^{\circ} \mathrm{C}$.

$R N A$ isolation and reverse transcription-quantitative $(R T-q)$ PCR amplification. RT-qPCR was performed as described previously (25). Briefly, total RNA was extracted using TRIzol ${ }^{\circledR}$ reagent (Thermo Fisher Scientific, Inc.) and was reverse transcribed by SuperScript ${ }^{\circledR}$ cDNA Synthesis Kit (Thermo Fisher Scientific, Inc.) according to the manufacturer's instructions. Briefly, an RT mixture containing $1 \mu 1$ total RNA, $4 \mu 1$ deoxynucleoside triphosphates, $2 \mu$ l Primer Max, $4 \mu \mathrm{l}$ RT bufer, $1 \mu \mathrm{l}$ SuperRT and diethyl pyrocarbonate (DEPC)-treated water to a final volume of $20 \mu \mathrm{l}$ were subjected to $37^{\circ} \mathrm{C}$ for $1 \mathrm{~h}, 95^{\circ} \mathrm{C}$ for $5 \mathrm{~min}$ and $4^{\circ} \mathrm{C}$ for cooling for $30 \mathrm{~min}$. The primers used were as follows: miR-217-5p forward, 5'-CGCGGATCCTATTGT ATTACGGTAGGATG-3' and reverse, 5'-CCGCTCGAGCAG ATAGCACGAACTTTT-3'; FGFR2 forward, 5'-GCGTCT CCAACGCCAAAGAGTCTTTCGTATATTATCAAAAT-3' and reverse, 5'-CAGTGAATTTTGATAATATACGAAAGA
CTCTTTGGCGTTG-3'; Pax 7 forward, 5'-AGCCGAGTG CTCAGAATCAA-3' and reverse, 5'-TCCTCTCGAAAGCCT TCTCC-3'; MyoD forward, 5'-CGACTGCCTGTCCAGCAT AG-3' and reverse, 5'-GGACACTGAGGGGTGGAGTC-3'; myosin heavy chain (MyHC) forward, 5'-TGCCAAGACCGT GAGGAATG-3' and reverse, 5'-AATGCATCACAGCTCCCG TG-3'; and GAPDH forward, 5'-GGGTGATGCTGGTGC TGAGTATGT-3' and reverse, 5'-AAGAATGGGAGTTGC TGTTGAAGTC-3'. Thermal cycling conditions were $2 \mathrm{~min}$ at $95^{\circ} \mathrm{C}$ followed by 40 cycles of $95^{\circ} \mathrm{C}$ for $15 \mathrm{sec}$ and $60^{\circ} \mathrm{C}$ for $15 \mathrm{sec}$ on a Real-Time PCR Detection System (Bio-Rad Laboratories, Inc.), GAPDH was used as an internal control to normalize target gene transcripts. Each sample was measured at least three times and the $2^{-\Delta \Delta \mathrm{Cq}}$ method (26) was used to assess relative levels of the mRNAs of the target genes.

Target gene prediction. TargetScan (version $7.21 \mathrm{www}$. targetscan.org,) and miRNA.org (version 22.1; http://www. microrna.org) were used for scanning the candidate targets of miR-217-5p. Basic information of miR-217-5p was submitted online and the potential targets of miR-217-5p were presented.

Luciferase reporter assay. The $\mathrm{miR}-217-5 \mathrm{p}$ mimics (5'-UAC UGCAUCAGGAACUGAUUGGC-3'), miR-217-5p antagomir (5'-GCCAAUCAGUUCCUGAUGCAGUA-3') NC-mimics (5'-UUCUCCGAACGUGUCACGUTT-3'; 5'-ACGUGACAC GUUCGGAGAATT-3') and NC-antagomir (5'-CAGUAC UUUUGUGUAGUACAA-3') were purchased from Thermo Fisher Scientific, Inc. The amplified miR-192-5p mimic sequence and miR-NC were transfected into the SkMSCs using Lipofectamine ${ }^{\circledR} 2000$ (Invitrogen; Thermo Fisher Scientific, Inc.) according to the manufacturer's instructions.

To identify the binding sequences and uniform resource locator, luciferase reporter assay was used. The miR-217-5p mimics, miR-NC, or miR-217-5p antagomir and the pRL-TK vector (Promega Corporation) carrying the mutant (mut) or wild-type (wt) FGFR2 3' untranslated region (3'-UTR) were co-transfected into SkMSCs using Lipofectamine ${ }^{\circledR} 2000$ (Invitrogen; Thermo Fisher Scientific, Inc.). Three days later, cells were lysed with the Dual-Glo ${ }^{\circledR}$ Reagent (Promega Corporation), and luciferase activity was measured using a Dual-Luciferase Reporter Assay System (Promega Corporation). The firefly luciferase activity was normalized to Renilla luciferase activity.

Western blot analysis. Protein was extracted using RIPA buffer with protease inhibitor (Sigma-Aldrich; Merck KGaA), protein concentration was determined by bicinchoninic assay (Thermo Fisher Scientific, Inc.), and denatured for $5 \mathrm{~min}$ at $100^{\circ} \mathrm{C}$ prior to electrophoresis using an $8-10 \%$ polyacrylamide gel and a mid-range protein ladder (Beijing CoWin Biotech Co., Ltd.). Then, proteins were transferred to a PVDF membrane (EMD Millipore), blocked with 2\% goat serum (Gibco; Thermo Fisher Scientific, Inc.) for $1 \mathrm{~h}$ at room temperature, and incubated with rabbit anti-FGFR2 (1:1,000; cat. no. ab10648; Abcam), GAPDH (1:1,000; EPR1689; cat. no. ab181602; Abcam), Pax7 (1:100; cat. no. ab199010; Abcam), MyoD (1:250; cat. no. ab203383; Abcam) and MHC (1:200; cat. no. ab11083; Abcam) primary antibodies overnight at $4^{\circ} \mathrm{C}$. Membranes were then washed using TBST $+0.5 \%$ Tween-20 (EMD Millipore) and incubated 
with Alexa Fluor ${ }^{\circledR} 790$-conjugated polyclonal goat anti-rabbit IgG H\&L (1:10,000; cat. no. ab175781; Abcam) secondary antibody at room temperature for $1 \mathrm{~h}$. Images were acquired by scanning with LI-COR's Odyssey Infrared Imaging System (LI-COR Biotechnology).

MTT assay. MTT assay was performed to evaluate the rate of proliferation of SkMSCs. Following transfection with miR-217-5p mimics, miR-217-5p antagomir and miR-NC, cells were seeded into 96 -well plates $\left(2 \times 10^{4}\right.$ cells/well) and incubated at $37^{\circ} \mathrm{C}$ for $\sim 24 \mathrm{~h}$. Then, $20 \mu \mathrm{l} 5 \mathrm{mg} / \mathrm{ml}$ MTT solution was added into each well, and the plates were incubated at $37^{\circ} \mathrm{C}$ for an additional $4 \mathrm{~h}$. After removing the medium, DMSO (160 $\mu \mathrm{l} /$ well) was added to each well. The concentration of MTT formazan solubilized with PBS was measured by a microplate reader (Tecan Group, Ltd.) at $490 \mathrm{~nm}$ according to the manufacturer's instructions.

FACS. Flow cytometry analysis and cell sorting were performed at the central laboratory of The First Affiliated Hospital of Sun Yat-sen University (Guangzhou, China). Markers for CD45 [BV510 mouse anti-rat CD45 Clone OX-1 (RUO); BD Pharmingen], CD11b (rat CD11B APC WT.5; BD Pharmingen), anti-integrin $\beta 1$ [anti-integrin $\beta 1$ (HMb1-1; PE/Cy7); cat. no. ab95622; Abcam] and CD34 [anti-CD34 antibody (EP373Y); cat. no. ab81289; Abcam] were used. The trypsinized cells were filtered using 200-mesh sieves and incubated with the above antibodies at $4^{\circ} \mathrm{C}$ for $1 \mathrm{~h}$. Then, the cells were washed twice with PBS and resuspended in $200 \mathrm{ml}$ PBS prior to analysis using a BD Accuri C6 flow cytometer (BD Biosciences) and flow cytometric data was analyzed using FlowJo software (version 7.6; Tree Star, Inc) according to the manufacturer's instructions.

Cell proliferation assay. To verify the proliferation of SkMSCs, a 5-Ethynyl-2'deoxyuridine (EdU) Kit (Guangzhou RiboBio Co., Ltd.) was used. SkMSCs were seeded at a density of $5 \times 10^{5}$ cells/well in 6-well plates coated with decellularized skeletal muscle extracellular matrix hydrogels (Shanghai Linbo Scientific Instruments Co., Ltd.) and cultured in the appropriate growth medium for $24 \mathrm{~h}$ at $37^{\circ} \mathrm{C}$ for the following four groups: Negative control (NC), miR217-5p, $2 \mu \mathrm{M}$ AZD4547 and $2 \mu \mathrm{M}$ miR217-5p + AZD4547. The cells were treated with $10 \mu \mathrm{M}$ EdU working solution growth medium for $2 \mathrm{~h}$ in the dark. Then, the cells were treated with PBS containing $4 \%$ paraformaldehyde for $20 \mathrm{~min}$ at room temperature, followed by $2 \mathrm{mg} / \mathrm{ml}$ glycine and $0.5 \%$ Triton X-100 for $15 \mathrm{~min}$ at room temperature. After Hoechst 33342 was added to each well, cells were incubated for 30-40 min in the dark. Images were acquired at $x 40$ magnification using an Axiovison 4.8 camera attached to an Axio Observer Z1 inverted microscope (Carl Zeiss, Inc.). Five image fields were randomly captured for each sample.

Immunofluorescence staining and microscopy. Immunofluorescence was performed as previously described (27). Samples were fixed with 4\% paraformaldehyde for $1 \mathrm{~h}$ at room temperature and then blocked with $2 \%$ goat serum (Gibco; Thermo Fisher Scientific, Inc.) for $1 \mathrm{~h}$ at room temperature. DAPI (cat. no. ab228549, Abcam) was used to identify the nuclei. The cells were incubated with primary antibodies overnight at $4^{\circ} \mathrm{C}$ and were as follows: Pax7 (1:100, cat. no. ab199010, Abcam), MyoD (1:250, cat. no. ab203383, Abcam) and MHC (1:200, cat. no. ab11083, Abcam). Then the cells were incubated with secondary antibodies for $1 \mathrm{~h}$ at room temperature and were as follows: Alexa Fluor ${ }^{\circledR}$ 594-conjugated goat anti-mouse (1:1,000, cat. no. ab150116, Abcam) and Alexa Fluor ${ }^{\circledR} 488$-conjugated goat anti-rabbit IgG H\&L (1:1,000, cat. no. ab150077, Abcam). Cell images were acquired using an Axiovison 4.8 camera (magnification, $x 40$ ) attached to an Axio Observer Z1 inverted microscope (Carl Zeiss, Inc.) and the images were then assembled using Adobe ${ }^{\circledR}$ Photoshop CS 6 software (Adobe Systems, Inc.).

Confocal microscopy. Cells (1x104 cells/well) were seeded on confocal dishes and maintained in an incubator for $24 \mathrm{~h}$ at $37^{\circ} \mathrm{C}$. The procedures were performed according to the manufacturer's instructions (Thermo Fisher Scientific, Inc.). Cells were incubated overnight at $4^{\circ} \mathrm{C}$ with anti- Pax7 (1:200; cat. no. ab199010; Abcam) and MyoD (1:200; cat. no. ab203383; Abcam) primary antibodies, and then incubated with Alexa Fluor ${ }^{\circledast}$ 594-conjugated goat anti-mouse $(1: 1,000$; cat. no. ab150116; Abcam) and Alexa Fluor ${ }^{\circledR} 488$-conjugated goat anti-rabbit IgG H\&L (1:1,000; cat. no. ab150077; Abcam) secondary antibodies for $1 \mathrm{~h}$ in the dark at room temperature. Then, DAPI was added for $15 \mathrm{~min}$ at room temperature. Finally, a confocal microscope (Nikon Corporation) was used to acquire images at $\mathrm{x} 40$ magnification.

Statistical analysis. Each experiment was repeated at least three times and the data are presented as the mean \pm standard deviation. Statistical significance was determined by performing Student's t-test for comparisons between two groups and one-way analysis of variance followed by Tukey's post-hoc test for comparisons between more than two groups. $\mathrm{P}<0.05$ was considered to indicate a statistically significant difference.

\section{Results}

Isolation and identification of SkMSCs. FACS analysis results demonstrated SkMSCs to be positive for CD34 and integrin $\beta 1$ and negative for CD11b and CD45 (Fig. 1A). Cell colonies were observed 3-5 days after the initial plating, and light microscopy demonstrated the SkMSCs exhibited a fibroblast-like morphology.

To identify the characteristics of SkMSCs, the expression of Pax7 and MyoD was examined. The majority of adhered SkMSCs exhibited expression of Pax7 and MyoD (Fig. 1B). In addition, the PG group exhibited a greater number of EdU-positive stained cells compared with that in the NC group (Fig. 1C and D), suggesting a high capacity for proliferation.

SkMSCs exhibiting an accelerated rate of proliferation express high levels of miR-217-5p. miR-217-5p levels were measured in the medium samples from PG and NC SkMSCs. RT-qPCR analysis demonstrated that the expression of miR-217-5p was significantly higher in PG SkMSCs compared with that in NC SkMSCs (Fig. 2A). In addition, the FGFR2 mRNA and protein expression was significantly upregulated in 
A

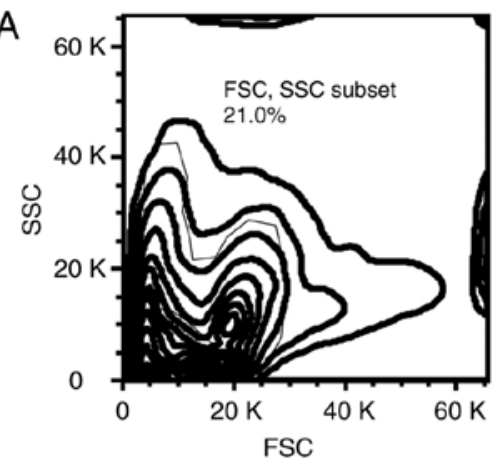

B

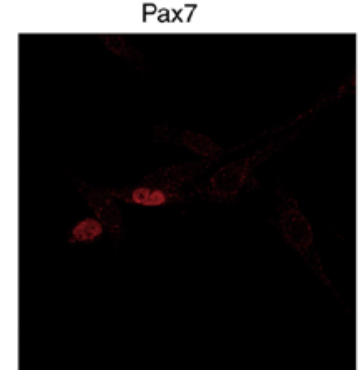

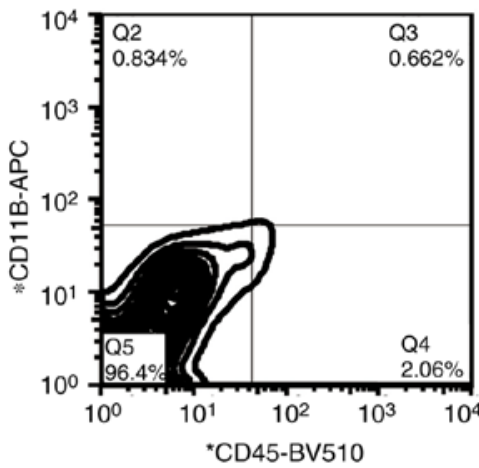

Myod

DAPI
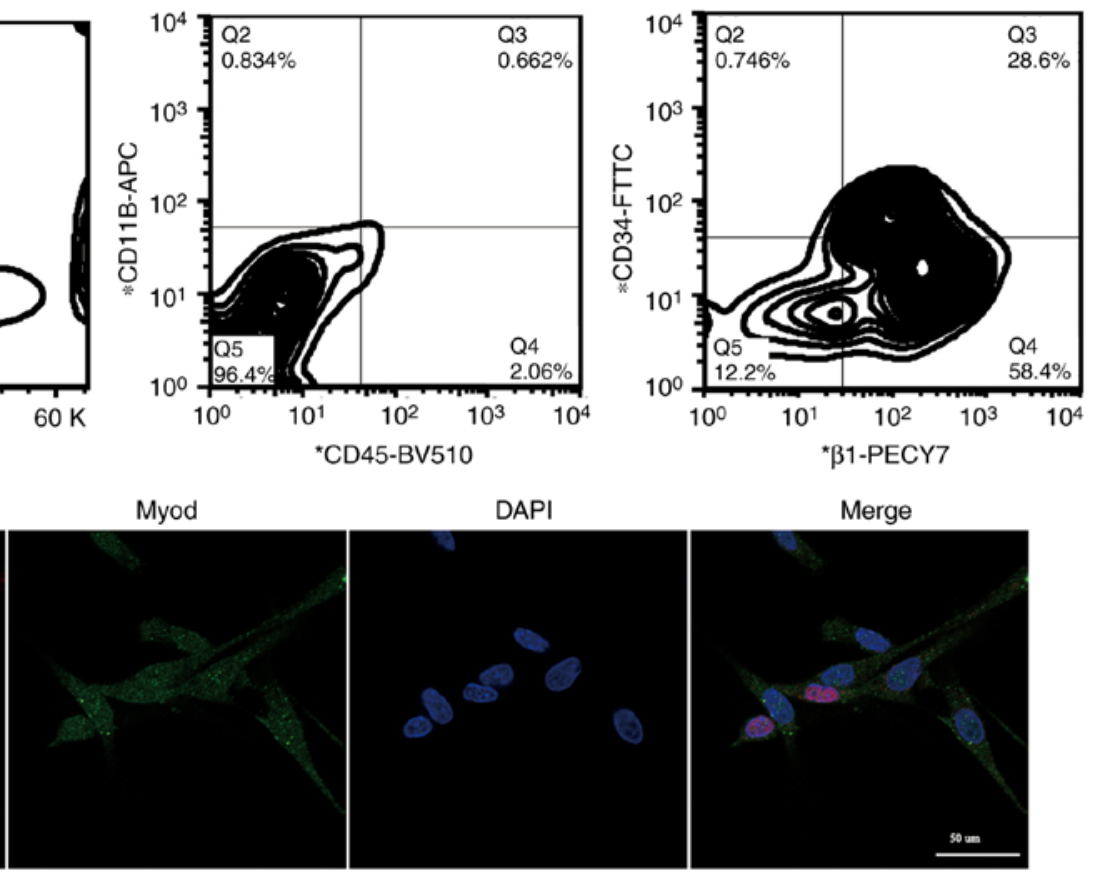

C

EDU

Hoechst
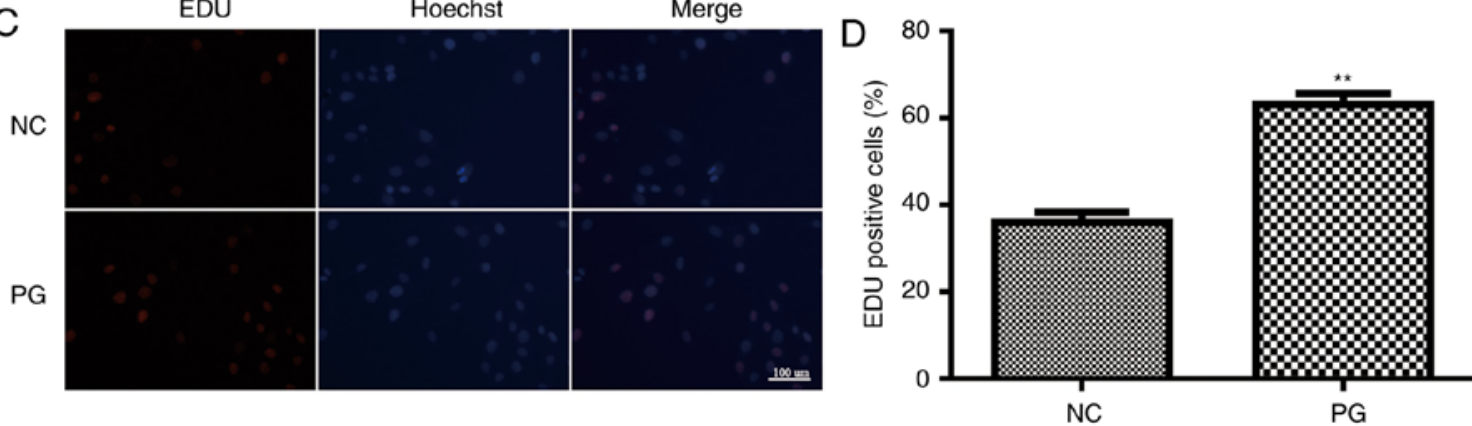

Figure 1. Isolation and identification of SkMSCs. (A) Double-sorted SkMSCs were positively stained with antibodies against CD34 and integrin $\beta 1$, but negative for CD11b and CD45. (B) Immunofluorescence staining images for Pax7 (red) and MyoD (green). Cell nuclei were stained by DAPI (blue). Scale bar, $10 \mathrm{~mm}$. (C) EdU assays demonstrated the proliferation of SkMSCs in the NC and PG groups. Scale bar, $50 \mathrm{~mm}$ (D) Quantification of EdU-positive cells in the NC and PG groups. NC, control group (SkMSCs cultured in DMEM supplemented with 10\% FBS); PG, high-proliferation group (SkMSCs cultured in DMEM supplemented with $20 \%$ FBS and $1 \%$ chick embryo extract with transforming growth factor $\beta 1)$. ${ }^{* *} \mathrm{P}<0.01$. SkMSCs, skeletal muscle stem cells; Pax7, paired box protein7 Pax7; MyoD, myogenic differentiation markers; EdU, 5-ethynyl-2'-deoxyuridine; NC, control group; PG, high-proliferation group.

PG SkMSCs compared with that in NC SkMSCs (Fig. 2B-D). These results suggested that miR-217-5p and FGFR2 expression was greater in highly proliferating SkMSCs compared with normal SkMSCs.

Ectopic expression of miR-217-5p affects SkMSC proliferation and differentiation. miR-217-5p expression was modulated by transfecting a miR-217-5p antagomir or miR-217-5p mimics into SkMSCs. MTT assay demonstrated that the proliferation of SkMSCs was promoted by miR-217-5p mimics and impeded by miR-217-5p antagomir compared with that of the miR-NC group (Fig. 3A). RT-qPCR analysis was also performed to verify miR-217-5p expression; miR-217-5p mimics significantly increased, whereas miR-217-5p antagomir significantly decreased the expression levels of miR-217-5p in SkMSCs compared with that of SkMSCs transfected with miR-NC (Fig. 3B). In addition, immunofluorescence staining demonstrated that the expression levels of MyoD and MYHC were significantly lower in the miR-217-5p mimic group and significantly higher in the miR-217-5p antagomir group compared with those of the miR-NC group (Fig. 3C-E). These data suggested that miR-217-5p enhanced the proliferation and inhibited the differentiation of SkMSCs.

FGFR2 is a direct target of miR-217-5p in SkMSCs. TargetScan and miRNA databases were used to predict the downstream targets of miR-217-5p and further explore the underlying molecular mechanism involved in the proliferation of SkMSCs. As binding between the 3'-UTR of FGFR2 and miR-217-5p was predicted (Fig. 4A), luciferase reporter assay was performed to investigate whether FGFR2 was directly targeted by miR-217-5p. Transfection of SkMSCs with miR-217-5p mimics significantly decreased the luciferase activity of the wild-type, but not the mutant, 3'-UTR of FGFR2 compared to that of SkMSCs transfected with miR-NC (Fig. 4B). By contrast, the miR-217-5p antagomir significantly increased the luciferase activity of wild-type FGFR2 in SkMSCs compared with that of SkMSCs transfected with miR-NC and miR-217-5p mimics (Fig. 4B). In addition, the results also demonstrated that miR-217-5p mimics 
A

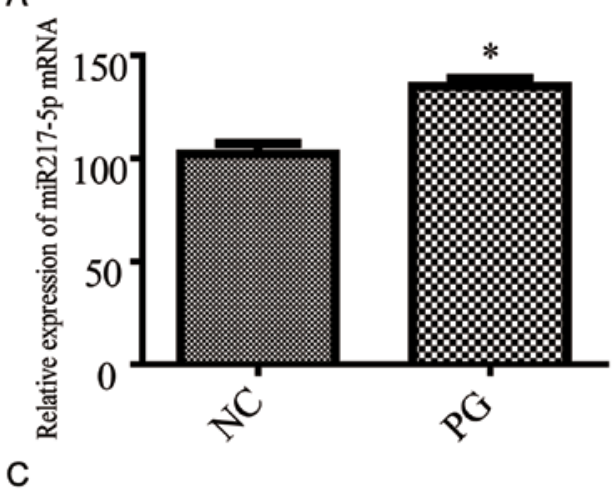

B
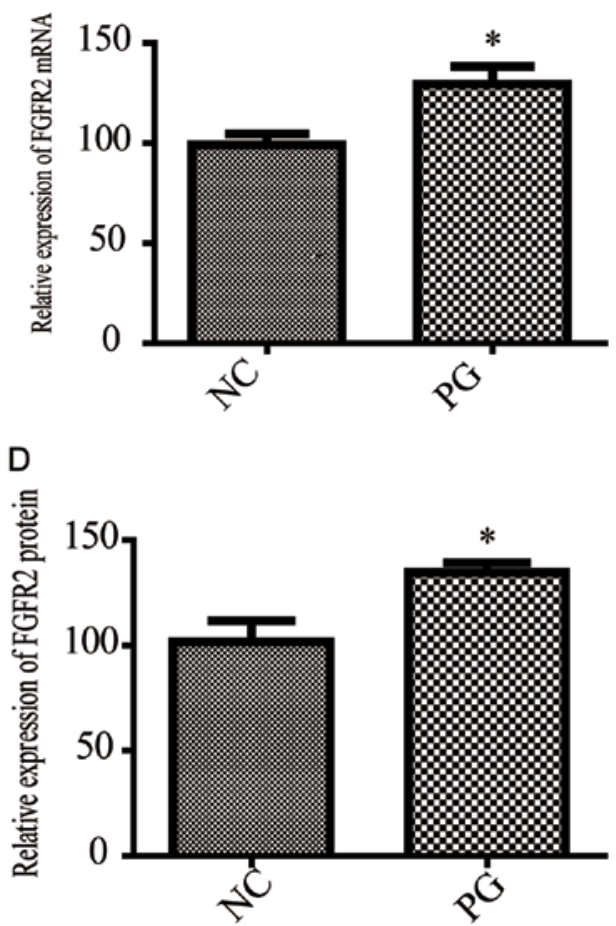

Figure 2. Upregulation of miR-217-5p and FGFR2 in accelerating proliferation of SkMSCs. (A) miR-217-5P expression in accelerating proliferation of SkMSCs. GAPDH mRNA was used as an internal control. (B) mRNA and (C and D) protein expression levels of FGFR2 were higher in accelerating proliferation of SkMSCs. GAPDH was used as an internal control. "P<0.05. miR, microRNA; NC, control group; PG, high-proliferation group; FGFR2, fibroblast growth factor receptor 2; SkMSCs, skeletal muscle stem cells.

significantly increased and miR-217-5p antagomir significantly decreased the mRNA and protein expression levels of FGFR2 in SkMSCs compared with those of miR-NC transfected SkMSCs (Fig. 4C-E). These findings suggested that FGFR2 was a direct target of miR-217-5p in SkMSCs.

$m i R-217-5 p$ regulates the proliferation and differentiation of SkMSCs by targeting FGFR2. To verify the mechanism of miR-217-5p in myogenesis, SkMSCs were transfected with miR-217-5p mimics or miR-NC. RT-qPCR results demonstrated that FGFR2 mRNA expression was increased in SkMSCs transfected with miR-217-5p mimics compared with that in SkMSCs transfected with miR-NC (Fig. 5A). In addition, RT-qPCR and western blotting results demonstrated that a selective FGFR inhibitor (AZD4547) suppressed the mRNA and protein expression of FGFR2 compared with that in the miR-NC group, whereas miR-217-5p overexpression (miR-217-5p mimics + AZD4547) reduced this suppression (Fig. 5A-C). Additionally, MTT assay demonstrated that the proliferation of SkMSCs was suppressed by AZD4547 compared with miR-NC, and that miR-217-5p mimics + AZD4547 reduced the reversal (Fig. 5D). Furthermore, to study the role of miR-192-5p in differentiation of SkMSCs, the expression of MYHC and MyoD was examined. Western blotting analysis demonstrated that the protein expression levels of MYHC and MyoD was suppressed by miR-217-5p mimics but enhanced by AZD4547 compared with those in the miR-NC group (Fig. 5E and F). These results suggested that miR-217-5p may regulate the myogenesis of SkMSCs by targeting FGFR2.

\section{Discussion}

The regenerative capacity of adult skeletal muscle is attributed to SkMSCs. Which have the ability to proliferate, differentiate and self-renew $(1,7)$. SkMSCs are involved in muscle formation and regeneration in response to acute or chronic injury $(7,28)$. The results of the present study demonstrated that the expression levels of miR-217-5p were increased in SkMSC culture medium and that miR-217-5p mimics promoted the proliferation and suppressed the differentiation of SkMSCs. In addition, miR-217-5p may have the potential to facilitate the proliferation of SkMSCs possibly by targeting FGFR2.

A miRNA is a type of small noncoding RNA 20-30 nucleotides in length that regulates gene expression through the inhibition of translation or promotion of the degradation of target mRNA by binding to its 3'-UTR (17,19,29-32). Recent studies have reported that the prerequisite for the myogenic differentiation of quiescent SkMSCs is the activation of myogenic markers and MyoD expression (7,33-35). Previous studies have reported that miRNAs are involved in regeneration and differentiation of SkMSCs (36-39). Increasing evidence indicates that several miRNAs promote or inhibit stem cell progression (19). miR-217-5p has been implicated in the apoptosis of colorectal cancer cells by directly targeting protein kinase c iota type I (PRKCI), BAG family molecular chaperone regulator 3 (BAG3), integrin subunit alpha $\mathrm{v}$ (ITGAV) and mitogen-activated protein kinase 1 (MAPK1) (19). Further studies have revealed that miR-217-5p regulates pluripotent stem cell proliferation and differentiation by LPS (17-19). However, the role of miR-217-5p in 
A

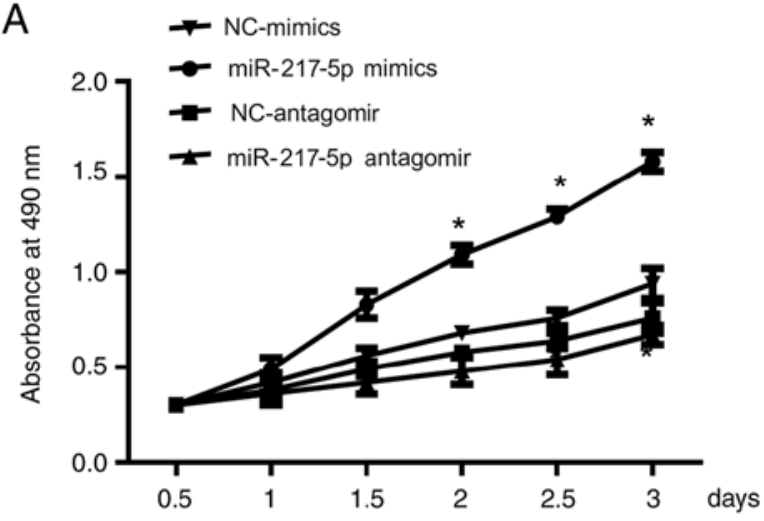

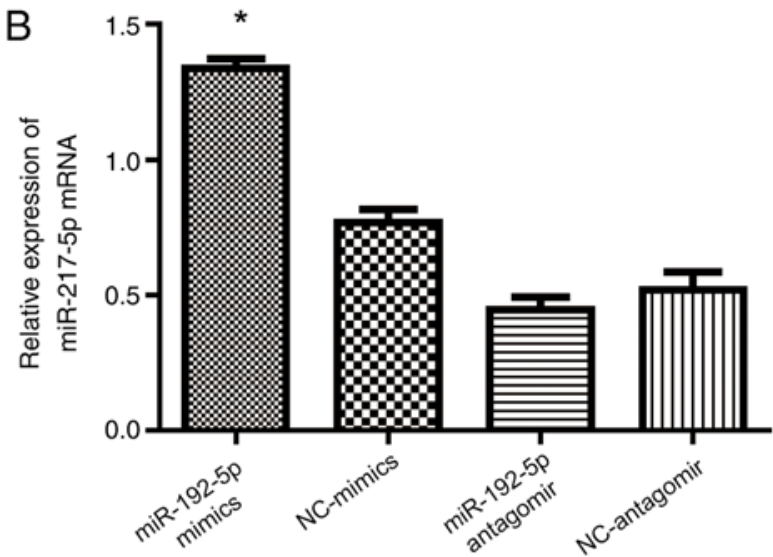

C

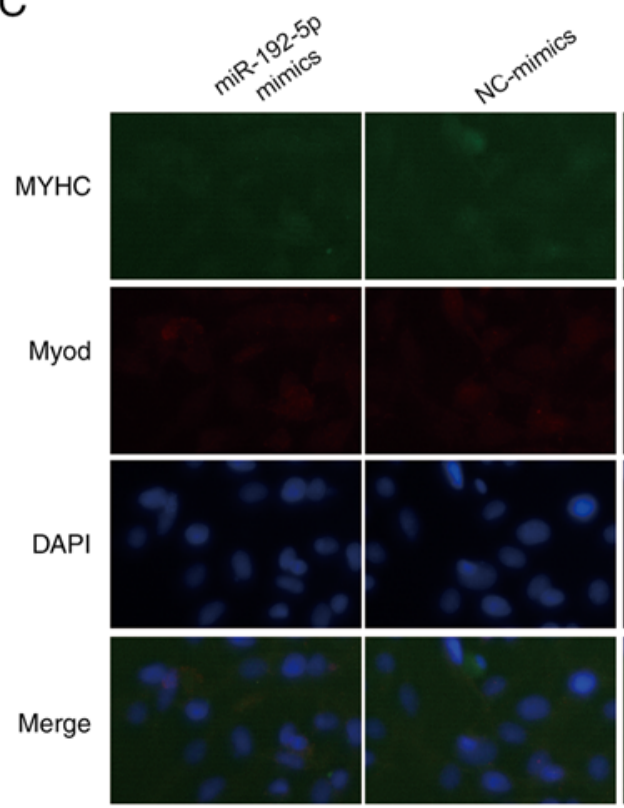

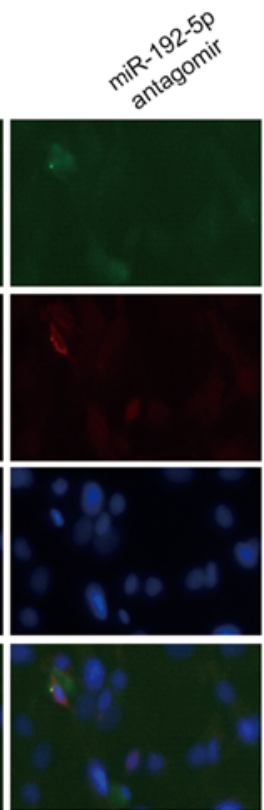

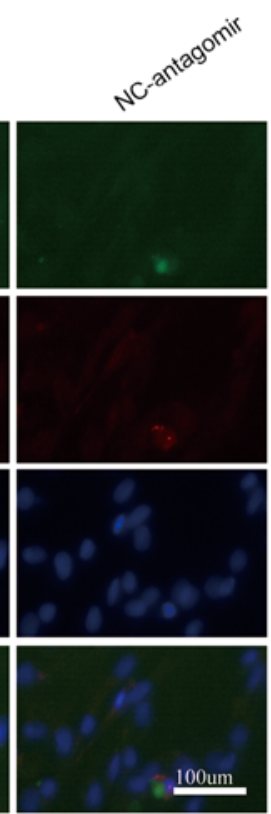

D miR-217-5p mimics

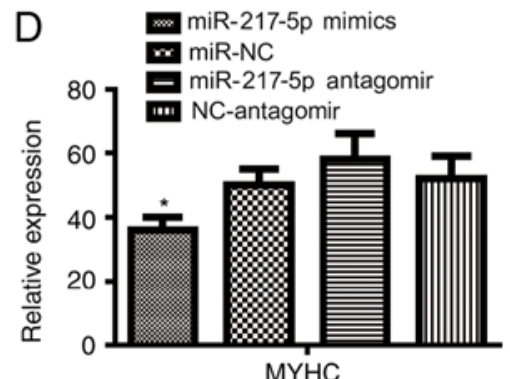

MYHC

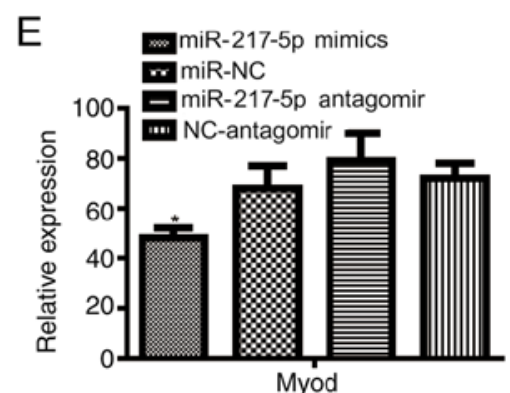

Figure 3. Ectopic expression of miR-217-5p affects the proliferation and differentiation of SkMSCs. (A) SkMSCs were transfected with NC-antagomir, NC-mimics, miR-217-5p antagomir and miR-217-5P mimic. MTT assay suggested that cell viability was enhanced upon miR-217-5p overexpression. (B) miR-217-5p expression was examined by RT-qPCR assay. (C) Immunofluorescence staining images of the expression of MyoD (red) and MYHC (green) in the three groups. (D and E) Quantification of MyoD and MYHC. "P $<0.05$. Scale bar, $100 \mu \mathrm{m} \mathrm{miR,} \mathrm{microRNA;} \mathrm{NC,} \mathrm{control} \mathrm{group;} \mathrm{SkMSCs,} \mathrm{skeletal} \mathrm{muscle}$ stem cells; RT-qPCR, reverse transcription-quantitative PCR; MyoD, myogenic differentiation markers; MyHC, myosin heavy chain.

SkMSCs is still unclear. The present study explored the effects of miR217-5p on the proliferation of SkMSCs. The results of the present study demonstrated that the expression levels of miR-217-5p were increased in SkMSC culture medium and promoted SkMSCs proliferation compared with that of the miR-NC group.

FGFR2 is associated with breast, lung and clear cell renal cell carcinomas (40-42). In addition, FGFR2 has recently been identified as a therapeutic target for carcinoma owing to its association with tumorigenesis (43-45). Studies have reported that FGFR2 promotes the proliferation of stem cells $(46,47)$ and that a novel circular RNA of FGFR2 may serve a role in enhancing skeletal muscle proliferation and differentiation by targeting miR-133a-5p and miR-29b-1-5p (6). A recent study has revealed that miR-142-3p suppresses the induction of the FGFR2-driven oncogenic process by directly binding transient receptor potential ankyrin-1 (TRPA1) (20).
The results of the present study suggested that miR-217-5p may directly target FGFR2 and enhance the expression of FGFR2, indicating a positive regulatory effect of miR-217-5p on this target gene. Although miRNAs have a predominantly negative effect on the expression of the protein encoded by the target gene, several reports have demonstrated a positive effect of miRNAs (48-51). Similarly, the results of the present study suggested that miR-217-5p promoted the target gene expression.

In the present study, TargetScan and miRNA databases were used to predict the downstream targets of miR-217-5p and further explore the molecular mechanism underlying the proliferation and differentiation of SkMSCs. SkMSCs have self-renewal properties and can regenerate muscle (28). However, a previous study has suggested that the SkMSC population contains undifferentiated cells that can differentiate into several other types of mesenchymal cells, such as 
A

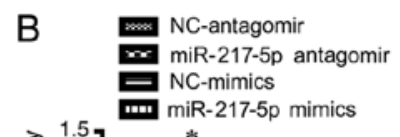

FGFR2 3' UTR ACUUACAGAGCACAAAUGCAGUA WT (9346-9352)

||||||

rno-miR-217-5p GGUCAGUCAAGGACUACGUCAU

FGFR2 3'UTR ACUUACAGAGCACAAUACGUCAA MUT
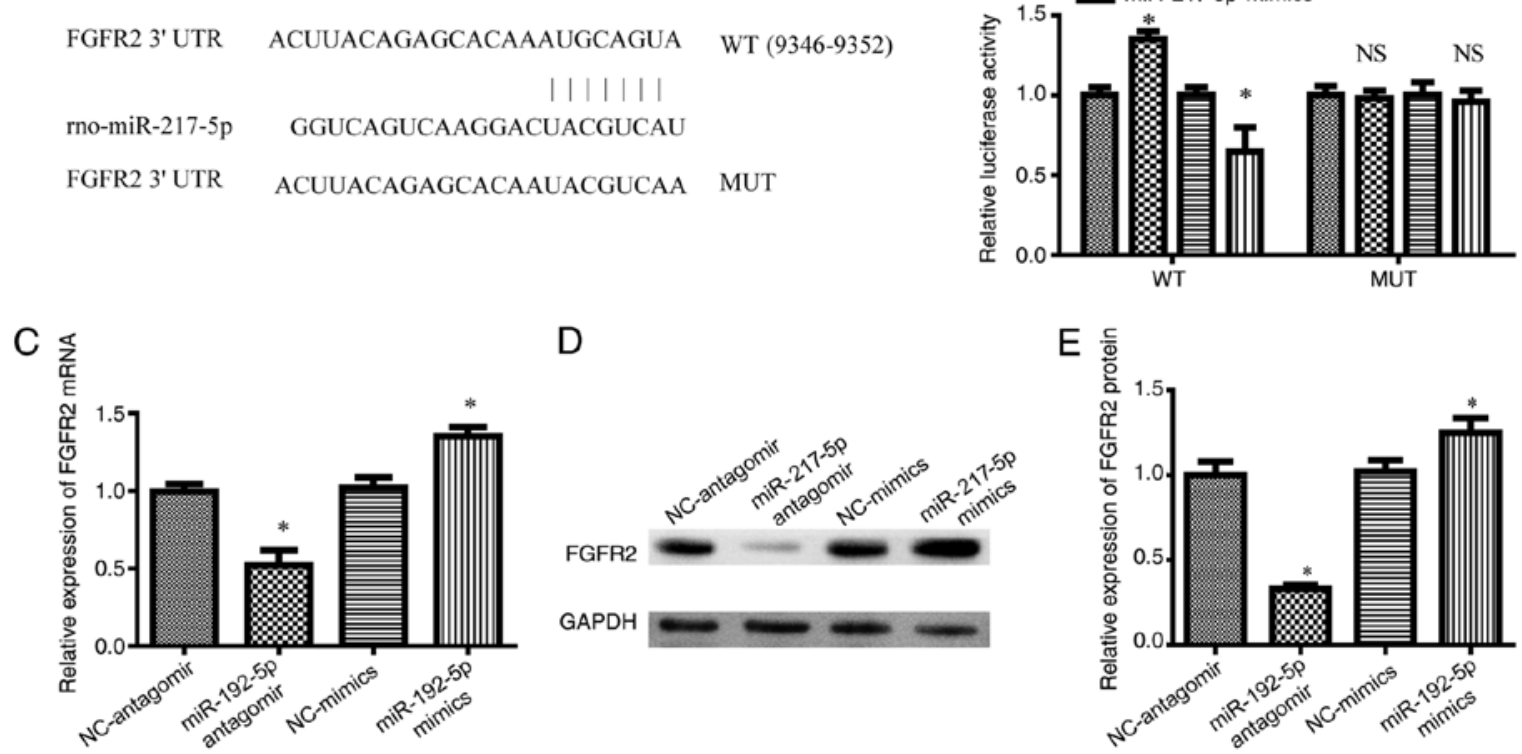

Figure 4. FGFR2 is a direct target of miR-217-5p. (A) Potential targets of miR-217-5p were identified in the 3'-UTR of FGFR2 through TargetScan. (B) Skeletal muscle stem cells transfected with the miR-217-5p mimic, NC-antagomir, NC-mimics or miR-192-5p antagomir in the presence of WT or MUT FGFR2 3'-UTR, and the binding was detected by measuring luciferase activity. (C-E) Reverse transcription-quantitative PCR and western blotting were performed to identify the (C) mRNA and (D and E) protein expression levels of FGFR2 in transfected cells. The level of FGFR2 was normalized to that of GAPDH. "P<0.05. miR, microRNA; NC, control group; UTR, untranslated region; FGFR2, fibroblast growth factor receptor 2; WT, wild-type; MUT, mutant.

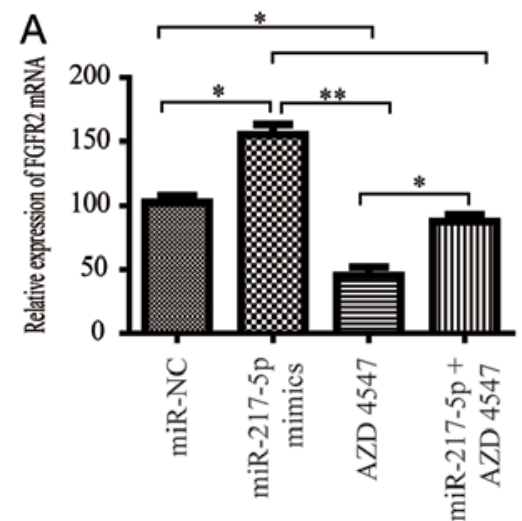

D

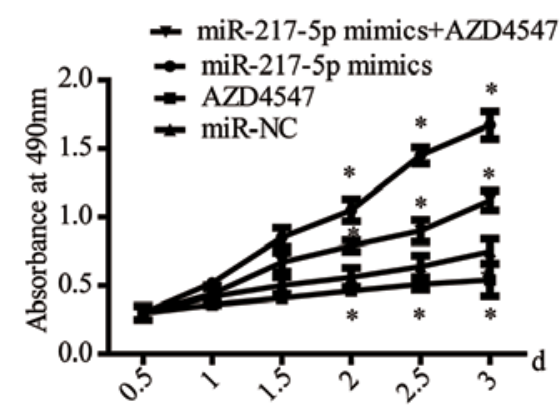

B

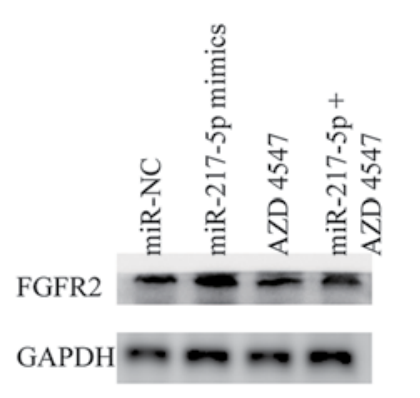

$\mathrm{E}$

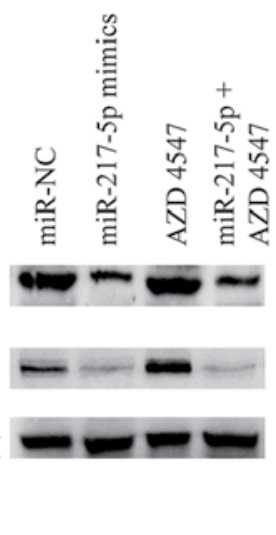

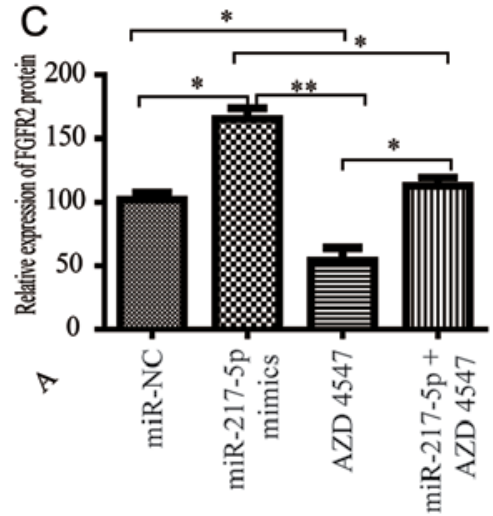

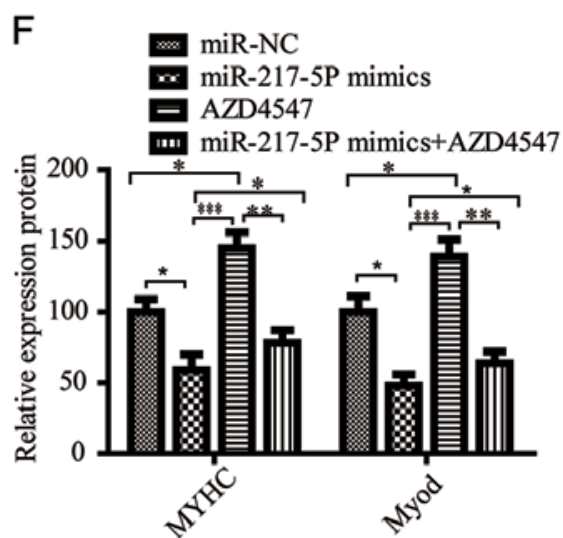

Figure 5. miR-217-5p regulates the proliferation and differentiation of SkMSCs by targeting FGFR2. (A-C) SkMSCs were transfected with miR-192-5p antagomir, miR-NC, miR-217-5p mimics or miR-217-5P mimics + AZD4547 and the (A) mRNA and (B and C) protein expression levels of FGFR2 were examined. The level of FGFR2 was normalized to that of GAPDH. (D) MTT assay results suggested that compared with that of the miR-NC group the proliferation of SkMSCs was suppressed by AZD4547, and miR-217-5p reduced this effect. (E and F) The protein expression levels of MYHC and MyoD was suppressed by miR-217-5p mimics but enhanced by AZD4547 compared with the miR-NC group. The level of MYHC and MyoD was normalized to that of GAPDH. " $\mathrm{P}<0.05,{ }^{, * *} \mathrm{P}<0.01,{ }^{* * *} \mathrm{P}<0.001$. miR, microRNA; NC, control group; SkMSCs, skeletal muscle stem cells; FGFR2, fibroblast growth factor receptor 2; MyoD, myogenic differentiation markers; MyHC, myosin heavy chain. 
adipocytes, chondrocytes and osteocytes (52). SkMSCs can be activated during muscle repair; however, they also have the potential to differentiate into other phenotypes. The results of the present study demonstrated that miR-217-5p mimics induced the upregulation of FGFR2, promoting myogenesis of SkMSCs.

There were several limitations to the present study. First, only one cell type was used in the present study, and additional cell types may be required. Second, although the present study indicated that miR-217-5p levels were increased in SkMSC culture medium, which promoted SkMSCs proliferation by targeting FGFR2, the underlying mechanism remained unclear. Third, the results of this study were not validated in vivo, necessitating further exploration of the role of miR-217-5p in SkMSCs. Further study to analyze the mechanism of skeletal muscle regeneration is necessary.

In summary, the results of the present study suggested that miR-217-5p maintains an appropriate proliferation rate and suppresses differentiation into a non-muscle cell phenotype, thus regulating the myogenesis of SkMSCs by targeting FGFR2, which may reflect a promising therapeutic strategy for the treatment of muscle injuries.

\section{Acknowledgements}

Not applicable.

\section{Funding}

This study was supported by The National Natural Science Foundation Item (grant nos. 81871787 and 81601057) and The Natural Science Foundation of Guangdong Province (grant nos. 2018A030310254 and 2015A030310350).

\section{Availability of data and materials}

The datasets used and/or analyzed during the present study are available from the corresponding author on reasonable request.

\section{Authors' contributions}

MZ and YY carried out experiments, data analysis and wrote the manuscript. BQ and GC performed experiments and helped with data quantifications. LG and JY designed the project and supervised the experiment. All authors read and approved the final manuscript.

\section{Ethics approval and consent to participate}

Animal care was approved by the Institutional Animal Care and Use Committee at The First Affiliated Hospital of Sun Yat-sen University (approval no. SYSU-IACUC-2020-000052).

\section{Patient consent for publication}

Not applicable.

\section{Competing interests}

The authors declare that they have no competing interests.

\section{References}

1. De Micheli AJ, Laurilliard EJ, Heinke CL, Ravichandran H, Fraczek P, Soueid-Baumgarten S, De Vlaminck I, Elemento O and Cosgrove BD: Single-cell analysis of the muscle stem cell hierarchy identifies heterotypic communication signals involved in skeletal muscle regeneration. Cell reports 30: 3583-3595.e5, 2020.

2. Sacco A, Doyonnas R, Kraft P, Vitorovic S and Blau HM: Self-renewal and expansion of single transplanted muscle stem cells. Nature 456: 502-506, 2008.

3. Sheehan SM and Allen RE: Skeletal muscle satellite cell proliferation in response to members of the fibroblast growth factor family and hepatocyte growth factor. J Cell Physiol 181: 499-506, 1999.

4. Cerletti M, Jurga S, Witczak CA, Hirshman MF, Shadrach JL, Goodyear LJ and Wagers AJ: Highly efficient, functional engraftment of skeletal muscle stem cells in dystrophic muscles. Cell 134: 37-47, 2008

5. Madaro L, Torcinaro A, De Bardi M, Contino FF, Pelizzola M, Diaferia GR, Imeneo G, Bouchè M, Puri PL and De Santa F: Macrophages fine tune satellite cell fate in dystrophic skeletal muscle of mdx mice. PLoS Genet 15: e1008408, 2019.

6. Chen X, Ouyang H, Wang Z, Chen B and Nie Q: A novel circular RNA generated by FGFR 2 gene promotes myoblast proliferation and differentiation by sponging miR-133a-5p and miR-29b-1-5p. Cells 7: E199, 2018.

7. Fukuda S, Kaneshige A, Kaji T, Noguchi YT, Takemoto Y, Zhang L, Tsujikawa K, Kokubo H, Uezumi A, Maehara K, et al: Sustained expression of HeyL is critical for the proliferation of muscle stem cells in overloaded muscle. Elife 8: e48284, 2019.

8. Tanaka KK, Hall JK, Troy AA, Cornelison DD, Majka SM and Olwin BB: Syndecan-4-expressing muscle progenitor cells in the SP engraft as satellite cells during muscle regeneration. Cell Stem Cell 4: 217-225, 2009.

9. Kumar P, Ciftci S, Barthes J, Knopf-Marques H, Muller CB, Debry C, Vrana NE and Ghaemmaghami AM: A composite Gelatin/hyaluronic acid hydrogel as an ECM mimic for developing mesenchymal stem cell derived epithelial tissue patches. J Tissue Eng Regen Med 14: 45-57, 2020.

10. Cao X, Tang S, Du F, Li H, Shen X, Li D, Wang Y, Zhang Z, Xia L, Zhu Q and Yin H: miR-99a-5p regulates the proliferation and differentiation of skeletal muscle satellite cells by targeting MTMR3 in chicken. Genes (Basel) 11: E369, 2020.

11. Yin H, He H, Shen X, Zhao J, Cao X, Han S, Cui C, Chen Y, Wei Y, Xia L, et al: miR-9-5p inhibits skeletal muscle satellite cell proliferation and differentiation by targeting IGF2BP3 through the IGF2-PI3K/Akt signaling pathway. Int J Mol Sci 21: E1655, 2020.

12. Fu L, Wang $\mathrm{H}$, Liao Y, Zhou P, Xu Y, Zhao Y, Xie S, Zhao S and Li X: miR-208b modulating skeletal muscle development and energy homoeostasis through targeting distinct targets. RNA Biol 17: 743-754, 2020.

13. Guan X, Gao Y, Zhou J, Wang J, Zheng F, Guo F, Chang A, Li X and Wang B: miR-223 regulates adipogenic and osteogenic differentiation of mesenchymal stem cells through a C/EBPs/miR-223/FGFR2 regulatory feedback loop. Stem Cells 33: 1589-1600, 2015.

14. Jedari B, Rahmani A, Naderi M and Nadri S: MicroRNA-7 promotes neural differentiation of trabecular meshwork mesenchymal stem cell on nanofibrous scaffold. J Cell Biochem 121: 2818-2827, 2020.

15. Phelps M, Stuelsatz P and Yablonka-Reuveni Z: Expression profile and overexpression outcome indicate a role for $\beta$ Klotho in skeletal muscle fibro/adipogenesis. FEBS J 283: 1653-1668, 2016.

16. Xiao Y, Guo Q, Jiang TJ, Yuan Y, Yang L, Wang GW and Xiao WF: miR4833p regulates osteogenic differentiation of bone marrow mesenchymal stem cells by targeting STAT1. Mol Med Rep 20: 4558-4566, 2019.

17. Zeng ZL, Lin XL, Tan LL, Liu YM, Qu K and Wang Z: MicroRNAs: Important regulators of induced pluripotent stem cell generation and differentiation. Stem Cell Rev Rep 14: 71-81, 2018.

18. Zhang X, Wang Z, Li W, Huang R, Zheng D and Bi G: MicroRNA-217-5p ameliorates endothelial cell apoptosis induced by ox-LDL by targeting CLIC4. Nutr Metab Cardiovase Dis 30: 523-533, 2020.

19. Flum M, Kleemann M, Schneider H, Weis B, Fischer S, Handrick R and Otte K: miR-217-5p induces apoptosis by directly targeting PRKCI, BAG3, ITGAV and MAPK1 in colorectal cancer cells. J Cell Commun Signal 12: 451-466, 2018.

20. Berrout J, Kyriakopoulou E, Moparthi L, Hogea AS, Berrout L, Ivan C,Lorger M, Boyle J,Peers C,Muench S, et al: TRPA1-FGFR2 binding event is a regulatory oncogenic driver modulated by miRNA-142-3p. Nat Commun 8: 947, 2017. 
21. Li X, Nie C, Tian B, Tan X, Han W, Wang J, Jin Y, Li Y, Guan X Hong $A$ and Chen $X$ : miR-671-5p blocks the progression of human esophageal squamous cell carcinoma by suppressing FGFR2. Int J Biol Sci 15: 1892-1904, 2019.

22. Nakano S, Nakamura K, Teramoto N, Yamanouchi K and Nishihara M: Basic fibroblast growth factor is pro-adipogenic in rat skeletal muscle progenitor clone, 2 G11 cells. Anim Sci J 87: 99-108, 2016

23. Du YE, Tu G, Yang G, Li G, Yang D, Lang L, Xi L, Sun K, Chen Y, Shu K, et al: MiR-205/YAP1 in activated fibroblasts of breast tumor promotes VEGF-independent angiogenesis through STAT3 signaling. Theranostics 7: 3972-3988, 2017.

24. Kwiatkowski BA, Kirillova I, Richard RE, Israeli D and Yablonka-Reuveni Z: FGFR4 and its novel splice form in myogenic cells: Interplay of glycosylation and tyrosine phosphorylation. J Cell Physiol 215: 803-817, 2008.

25. Zhu M, Liu C, Li S, Zhang S, Yao Q and Song Q: Sclerostin induced tumor growth, bone metastasis and osteolysis in breast cancer. Sci Rep 7: 11399, 2017.

26. Livak KJ and Schmittgen TD: Analysis of relative gene expression data using real-time quantitative PCR and the 2(-Delta Delta C(T)) method. Methods 25: 402-408, 2001.

27. Zou P, Zhu M, Lian C, Wang J, Chen Z, Zhang X, Yang Y, Chen X, Cui X, Liu J, et al: miR-192-5p suppresses the progression of lung cancer bone metastasis by targeting TRIM44. Sci Rep 9: 19619, 2019.

28. Schultz SS and Lucas PA: Human stem cells isolated from adult skeletal muscle differentiate into neural phenotypes. J Neurosci Methods 152: 144-155, 2006.

29. Chu Q, Sun Y, Bi D, Cui J and Xu T: Up-regulated of miR-8159-5p and miR-217-5p by LPS stimulation negatively co-regulate TLR1 in miiuy croaker. Dev Comp Immunol 67: 117-125, 2017.

30. Erdos Z, Barnum JE, Wang E, DeMaula C, Dey PM, Forest T, Bailey WJ and Glaab WE: Evaluation of the relative performance of pancreas specific microRNAs in rat plasma as biomarkers of pancreas injury. Toxicol Sci 173: 5-18, 2019.

31. Du W, Tang H,Lei Z,Zhu J,Zeng Y,Liu Zand Huang JA: miR-335-5p inhibits TGF- $\beta 1$-induced epithelial-mesenchymal transition in non-small cell lung cancer via ROCK1. Respir Res 20: 225, 2019.

32. Choi YJ, Kim H, Kim JW, Song CW, Kim DS, Yoon S and Park HJ: Phthalazinone pyrazole enhances the hepatic functions of human embryonic stem cell-derived hepatocyte-like cells via suppression of the epithelial-mesenchymal transition. Stem Cell Rev Rep 14: 438-450, 2018

33. Schmidt M, Schüler SC, Hüttner SS, von Eyss B and von Maltzahn J: Adult stem cells at work: Regenerating skeletal muscle. Cell Mol Life Sci 76: 2559-2570, 2019.

34. Dumont NA, Bentzinger CF, Sincennes MC and Rudnicki MA: Satellite cells and skeletal muscle regeneration. Compr Physiol 5: $1027-1059,2015$

35. Feige P, Brun CE, Ritso $M$ and Rudnicki MA: Orienting muscle stem cells for regeneration in homeostasis, aging, and disease Cell Stem Cell 23: 653-664, 2018

36. Sato T, Yamamoto T and Sehara-Fujisawa A: miR-195/497 induce postnatal quiescence of skeletal muscle stem cells. Nat Commun 5 : 4597, 2014.

37. Tirone M, Giovenzana A, Vallone A, Zordan P, Sormani M, Nicolosi PA, Meneveri R, Gigliotti CR, Spinelli AE, Bocciardi R, et al: Severe heterotopic ossification in the skeletal muscle and endothelial cells recruitment to chondrogenesis are enhanced by monocyte/macrophage depletion. Front Immunol 10: 1640, 2019.

38. Zhao Y, Chen M, Lian D, Li Y, Li Y, Wang J, Deng S, Yu K and Lian Z: Non-coding RNA regulates the myogenesis of skeletal muscle satellite cells, injury repair and diseases. Cells 8: E988, 2019.
39. Kletukhina S, Neustroeva O, James V, Rizvanov A and Gomzikova M: Role of mesenchymal stem cell-derived extracellular vesicles in epithelial-mesenchymal transition. Int J Mol Sci 20: E4813, 2019.

40. Sadej R, Lu X, Turczyk L, Novitskaya V, Lopez-Clavijo AF, Kordek R, Potemski P, Wakelam MJO, Romanska-Knight H and Berditchevski F: CD151 regulates expression of FGFR2 in breast cancer cells via PKC-dependent pathways. J Cell Sci 131: jes220640, 2018

41. Li L, Zhang S, Wei L, Wang Z, Ma W, Liu F and Qian Y: FGF2 and FGFR2 in patients with idiopathic pulmonary fibrosis and lung cancer. Oncol Lett 16: 2490-2494, 2018.

42. Vanmechelen M, Lambrechts D, Van Brussel T, Verbiest A, Couchy G, Schöffski P, Dumez H, Debruyne PR, Lerut E, Machiels JP, et al: Fibroblast growth factor receptor-2 polymorphism rs2981582 is correlated with progression-free survival and overall survival in patients with metastatic clear-cell renal cell carcinoma treated with sunitinib. Clin Genitourin Cancer 17: e235-e246, 2019

43. Maehara O, Suda G, Natsuizaka M, Ohnishi S, Komatsu Y, Sato F, Nakai M, Sho T, Morikawa K, Ogawa K, et al: Fibroblast growth factor-2-mediated FGFR/Erk signaling supports maintenance of cancer stem-like cells in esophageal squamous cell carcinoma. Carcinogenesis 38: 1073-1083, 2017.

44. Wang D, Yang L, Yu W and Zhang Y: Investigational fibroblast growth factor receptor 2 antagonists in early phase clinical trials to treat solid tumors. Expert Opin Investig Drugs 28: 903-916, 2019.

45. Wang Y and Qin H: miR-338-3p targets RAB23 and suppresses tumorigenicity of prostate cancer cells. Am J Cancer Res 8: 2564-2574, 2018.

46. Shanmuganathan $\mathrm{S}$ and Angayarkanni N: Chebulagic acid and Chebulinic acid inhibit TGF- $\beta 1$ induced fibrotic changes in the chorio-retinal endothelial cells by inhibiting ERK phosphorylation. Microvasc Res 121: 14-23, 2019.

47. Xu Y, Xiao H, Luo H, Chen Y, Zhang Y, Tao L, Jiang Y, Chen Y and Shen X: Inhibitory effects of oxymatrine on TGF- $\beta 1$-induced proliferation and abnormal differentiation in rat cardiac fibroblasts via the p38MAPK and ERK1/2 signaling pathways. Mol Med Rep 16: 5354-5362, 2017.

48. Huang V, Place RF, Portnoy V, Wang J, Qi Z, Jia Z, Yu A, Shuman M, Yu J and Li LC: Upregulation of Cyclin B1 by miRNA and its implications in cancer. Nucleic Acids Res 40: 1695-1707, 2012

49. Vasudevan S: Posttranscriptional upregulation by microRNAs. Wiley Interdiscip Rev RNA 3: 311-330, 2012.

50. Lu H, Buchan RJ and Cook SA: MicroRNA-223 regulates Glut4 expression and cardiomyocyte glucose metabolism. Cardiovasc Res 86: 410-420, 2010

51. Saifi M, Yogindran S, Nasrullah N, Nissar U, Gul I and Abdin MZ: Co-expression of anti-miR319g and miRStv_11 lead to enhanced steviol glycosides content in Stevia rebaudiana. BMC Plant Biol 19: 274, 2019.

52. Alessandri G, Pagano S, Bez A, Benetti A, Pozzi S, Iannolo G, Baronio M, Invernici G, Caruso A, Muneretto C, et al: Isolation and culture of human muscle-derived stem cells able to differentiate into myogenic and neurogenic cell lineages. Lancet 364 : 1872-1883, 2004

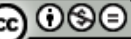

This work is licensed under a Creative Commons Attribution-NonCommercial-NoDerivatives 4.0 International (CC BY-NC-ND 4.0) License. 